\title{
Modeling and signal processing approaches for SAW chemical sensors
}

Fischerauer, Gerhard

Lehrstuhl für Mess- und Regeltechnik

Universität Bayreuth, 95440 Bayreuth, Germany

\section{Introduction}

Microacoustic chemical sensors based, for instance, on quartz crystal microbalances (QCM) or surface acoustic wave (SAW) devices have been popular in laboratories around the world for almost 30 years. They have also met with some commercial success, and one of the underlying effects, viz., mass loading, is routinely used in thin-film technology to monitor film growth during deposition. While hundreds of man-years have been spent on models and design software for SAW communications devices - an effort that has much reduced the effective development time for custom-specific devices in comparison with older trial and error strategies -, the same cannot be said about SAW sensors.

The difference in the modeling needs for communications and sensing devices is illustrated in Fig. 1 for the case of a delay line. The device consists of a piezoelectric substrate with two interdigital transducers (IDT) acting as electroacoustic antennas (Fig. 1a). When using the device as a bandpass filter, one exploits the frequency-selective conversion of the electric input signal $s_{\mathrm{el}}(t)$ into an acoustic signal $s_{\mathrm{ac}}(t)$ and the reconversion into an electric output signal $s_{e l}^{\prime}(t)$. Interfering quantities such as temperature or pressure or, if the device is not hermetically sealed, the ambient gas concentration $c_{a}$ may disturb the SAW propagation between the IDTs and thus influence the output signal $s_{\mathrm{el}}^{\prime}(t)$ (Fig. 1b). In a chemical sensor, this interference is the desired phenomenon: one is not interested in the action path from $s_{\mathrm{el}}(t)$ to $s_{\mathrm{el}}^{\prime}(t)$, but in the modulation of a signal feature of $s_{\mathrm{el}}^{\prime}(t)$ by the ambient gas concentration $c_{\mathrm{a}}(t)$ and therefore attempts to enhance this modulation by, e. g., attracting analyte molecules via a sensor layer deposited between the IDTs. A suitable signal feature is the SAW velocity, which is associated with the phase of $s_{\mathrm{el}}^{\prime}(t)$ and may be converted to a frequency $f_{\mathrm{m}}$ by inserting the SAW delay line in the feedback path of a two-port oscillator (Fig. 1c).

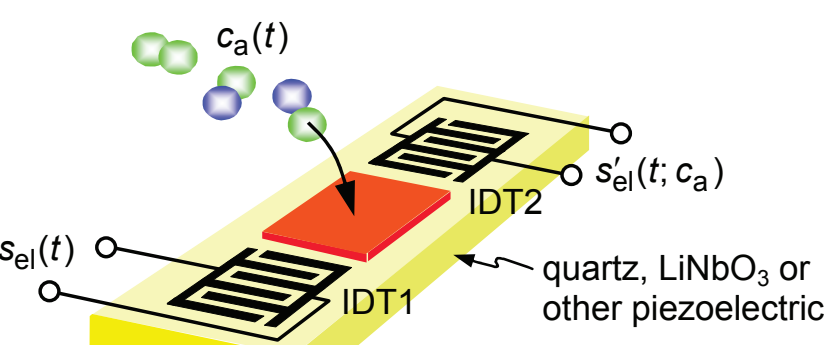

a)

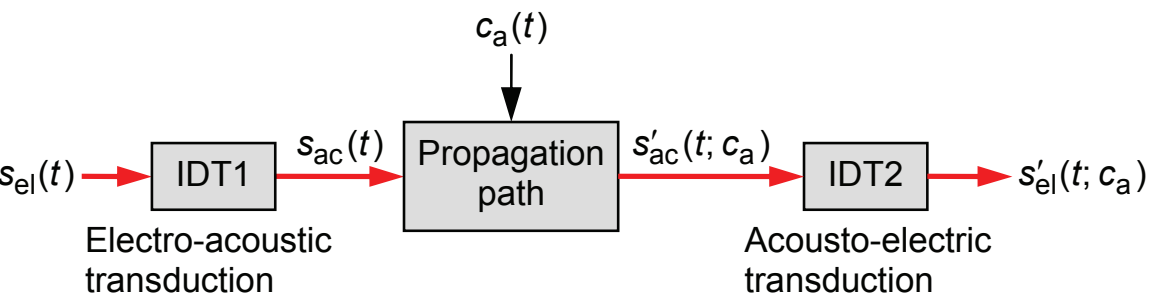

b) transduction

Acousto-electric

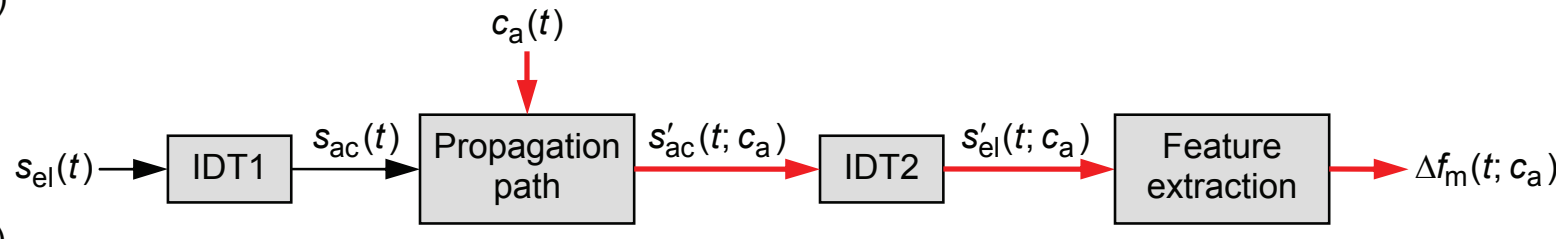

c)

Fig. 1: SAW delay line comprising two IDTs. a) Device layout. b, c) Action paths when the device is used b) as a bandpass filter and c) as a chemical sensor. 
From this, it follows that the key modeling problem for SAW chemical sensors involves the physical and functional aspects associated with the conversion of analyte concentration to acoustic wave properties and further on to device terminal characteristics. This contribution describes the state-of-the-art methods used to accomplish this and addresses some signal processing approaches capable of improving sensor system characteristics.

\section{Modeling of SAW device characteristics \\ 2.1 Numerical approaches}

The undisturbed SAW propagation along the surface of a piezoelectric solid is described by Newton's second law in its continuum mechanical form and the electrostatic part of Maxwell's equations together with the constitutive equations (material laws) and appropriate boundary conditions such as vanishing normal stress on a free surface. This results in a set of four partial differential equations (PDE) for the three mechanical particle displacement components $u_{i}(x, y, z, t)$ in the solid $(i=x, y, z)$ and the electrostatic potential $\varphi(x, y, z, t)[1]$.

By now, it has become a routine task to solve this set of coupled PDEs by discretizing the geometry and solving the equations on the discretized domain (Fig. 2). The method of choice is the Finite Element (FE) method, and both commercial software packages such as Ansys and proprietary programs are widely used in the SAW industry.

The decisive advantage of FE calculations is their flexibility; they can handle almost arbitrary geometries and deal not only with undisturbed SAW propagation, but also with excitation, detection, and scattering problems. The most striking disadvantages are the required computation times and the fact that many, possibly uncertain material parameters enter into the simulation. For instance, calculations for quartz sensors require the knowledge of 11 parameters for the quartz substrate (six stiffness tensor components, two permittivity tensor components, two piezoelectric tensor components, and the mass density) plus the analogous parameters of the sensitive layer. While single-crystal quartz is very well known [1, Vol. I, pp. $366 \mathrm{ff}$.] and many sensitive layers can be treated as isotropic, which reduces the number of material parameters to three (mass density and Lamé constants), one can only simulate a sensor response if one knows how the material parameters change with analyte gas concentration. The latter requirement is almost never fulfilled.

Nevertheless, FE calculations can be useful to deduce basic functional relationships. If one assumes that molecules incorporated in a sensor layer only contribute to the layer mass (mass loading), but do not change the elastic properties of the layer, the sensor effect can be modeled by either increasing the mass density in the layer or by increasing the layer height and keeping the mass density constant. An example for the second approach is shown in Fig. 3. This reproduces the well-known fact that mass loading decreases the SAW velocity, and that the fractional velocity shift is proportional to the mass increase if the latter remains small (Sauerbrey equation, [2]).

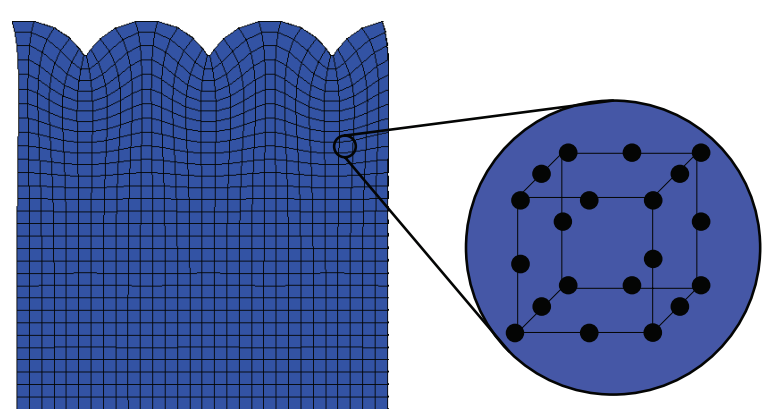

Fig. 2: Discretized geometry of a SAW device substrate showing a basic element (SOLID226 in Ansys) with 20 nodes and four unknowns ("degrees of freedom") per node $\left(u_{x}, u_{y}, u_{z}, \varphi\right)$. The values of the unknowns between the nodes are determined by interpolating with quadratic form functions. 


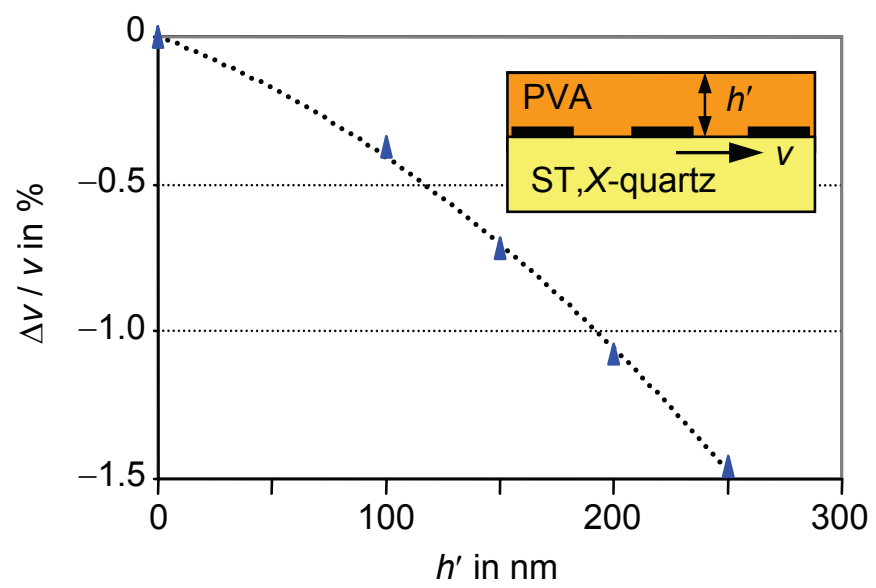

Fig. 3: FE calculation of the fractional velocity shift for a SAW on ST,X-quartz with a polymer (PVA) coating of thickness $h^{\prime}$. Polymer data: Lamé constants $\lambda^{\prime}=2.88 \mathrm{GPa}, \mu^{\prime}=0.26 \mathrm{GPa}$; mass density $\rho^{\prime}=1294 \mathrm{~kg} / \mathrm{m}^{3}$. IDT center frequency: $433.9 \mathrm{MHz}$. After [3].

\subsection{Analytical approaches}

At least in the case of undisturbed SAW propagation and simple geometries, the fundamental coupled PDEs can also be solved analytically. The partial-wave method transforms the PDEs into an algebraic eigenvalue problem by using trial functions in the form of a uniform plane wave $\left(u_{i}=\hat{u}_{i} \mathrm{e}^{\mathrm{j}(\omega t-\vec{k} \circ \vec{r})}\right.$, $\left.\varphi=\hat{\varphi} e^{\mathrm{j}(\omega t-\vec{k} \cdot \vec{r})}\right)$ and looking iteratively for the unique linear superposition of the resulting four eigensolutions that satisfies the boundary conditions. While the method is fast and straightforward, it lacks the generality of the FE method - e. g., it is cumbersome to include the effects of IDTs on the substrate surface. Moreover, it requires the knowledge of the same (often uncertain) material parameters and their dependence on the analyte gas concentration as the FE method. For this reason, the partial-wave method is mostly restricted to fundamental investigations like the search for propagation directions in which SAWs propagate along a free surface without being affected too much by temperature effects.

The description of the coupling between the acoustic waves and the electrical input and output signals is one of the standard tasks in connection with communications devices. For this reason, there exist a number of well-established methods. The most fundamental ones of these are

- the delta-function model, which constructs the normalized frequency dependence of the electroacoustic transfer function by assuming that each finger overlap is the source or sink of an acoustic wave packet and by then superposing these wave packets [4, pp. $57 \mathrm{ff}$.];

- the P-matrix model, which divides an IDT into homogeneous sections such as free surface, IDT finger edge or metalized surface, the effects of which are described by appropriate scattering matrices which are then cascaded to obtain the scattering matrix of the entire IDT [5]; and

- equivalent-circuit models such as the one which considers the IDT as electroacoustic antenna and models it by the shunt configuration of a capacitor (corresponding to the static IDT capacitance), a frequency-dependent resistor, and a frequency-dependent reactance, or the Butterworth-van Dyke model which describes a QCM or SAW resonator by lumped elements [6, pp. $389 \mathrm{ff}$.].

In contrast to the FE and the partial-wave methods, these approaches do not rely on the elementary material parameters (tensors), but instead incorporate easily measured characteristics such as the SAW propagation velocity, the propagation attenuation, the reflection coefficient at a discontinuity, or the resonance frequency of a device. The state of the art in the design of highly sophisticated communications devices is to include second-order effects in the device simulation (diffraction, electromagnetic feedthrough, etc.). For chemical sensors, this is less relevant as the details of the frequency response are of minor interest in comparison with the sensitivity of the signal features to the analyte gas concentration. Therefore, there exists almost no literature on the design of SAW chemical sensors mentioning the above methods. The literature is rather limited to general considerations regarding the device type (delay line, resonator), the operating frequency, and layout details (housing etc.). 


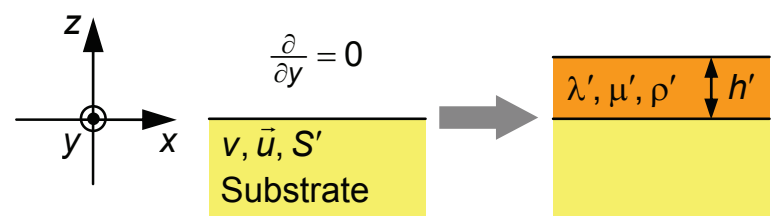

Fig. 4: Perturbation of an $x$-propagating SAW by a thin-film overlay with Lamé constants $\lambda^{\prime}$ and $\mu^{\prime}$, mass density $\rho^{\prime}$, and thickness $h^{\prime}$. Cf. text for explanation.

From the viewpoint of chemical sensors, the most useful analytical method consists in accepting the basic characteristics of a SAW device and determining the effects of small changes in device parameters such as coating layer thickness or density. Such a perturbation approach may, e. g., be applied to the case shown in Fig. 4. Initially, a SAW is propagating in $x$-direction on a free surface with velocity $v$ and intensity (power density per unit width, in $\mathrm{W} / \mathrm{m}) S^{\prime}$. At the surface $(z=0)$, the wave causes a particle displacement $\vec{u}=\left(u_{x}, u_{y}, u_{z}\right)^{\top}$ and a particle speed $\dot{\vec{u}}$. The deposition of a thin film on top of the substrate will change the wave velocity by $\Delta v$, and perturbation theory predicts this change to be [7; 1 , Vol. II, p. 277]

$$
\frac{\Delta v}{v}=-\frac{h^{\prime}}{4 v S^{\prime}}\left(\left(\rho^{\prime} v^{2}-\frac{4 \mu^{\prime}\left(\lambda^{\prime}+\mu^{\prime}\right)}{\lambda^{\prime}+2 \mu^{\prime}}\right) \cdot \dot{u}_{x}^{2}+\left(\rho^{\prime} v^{2}-\mu^{\prime}\right) \cdot \dot{u}_{y}^{2}+\rho^{\prime} v^{2} \cdot \dot{u}_{z}^{2}\right)
$$

In the case of pure mass loading (no elastic effects), this Tiersten formula reduces to

$$
\frac{\Delta v}{v}=-\frac{1}{4} v \cdot \rho^{\prime} h^{\prime} \cdot \frac{\dot{u}^{2}}{S^{\prime}}=-c_{m} f \cdot \rho^{\prime} h^{\prime}
$$

where use has been made of the fact that the quantity $\dot{u}^{2} / S^{\prime}$ is proportional to the operating frequency $f$ [1] and $c_{m}$ is a positive constant. Hence, the SAW velocity is a linear function of the accumulated mass per area $\left(\rho^{\prime} h^{\prime}\right)$, in line with the numerical prediction of Fig. 3 for small enough values of $h^{\prime}$, and the slope $\partial(\Delta v / v) / \partial\left(\rho^{\prime} h^{\prime}\right)$ is the steeper, the higher the operating frequency. The latter effect has been known to be true for QCMs since the 1950s [2].

Perturbation formulas for many cases such as mechanical loading, viscoelastic loading, electrical loading, and fluid loading and for many wave types may be found in the literature $[8,9]$.

\subsection{System-level approaches}

None of the methods discussed above considers the time response of a SAW chemical sensor. In other words, they are all restricted to the steady-state case. To describe the action path from the analyte gas concentration $c_{\mathrm{a}}(t)$ to the observed signal feature - say, a SAW resonator frequency $f_{\mathrm{m}}\left(t ; c_{\mathrm{a}}\right)-($ Fig. 1c), one must consider the details of the incorporation of molecules in the sensitive layer (Fig. 5). In many cases, the molecule diffusion is the dominant process and the reaction of the molecules at the binding sites can be neglected in comparison [10]. The solution of the reaction-diffusion equations under this assumption leads to a linear time-invariant $(\mathrm{LTI})$ model for the sensor with $c_{\mathrm{a}}(t)$ as input quantity and $f_{\mathrm{m}}(t)$ as output quantity [11]. Therefore, the complete theory of LTI systems may be applied to SAW chemical sensors. In particular, the response of the sensor to concentration steps or to arbitrary time-varying gas concentrations can be written in closed form.

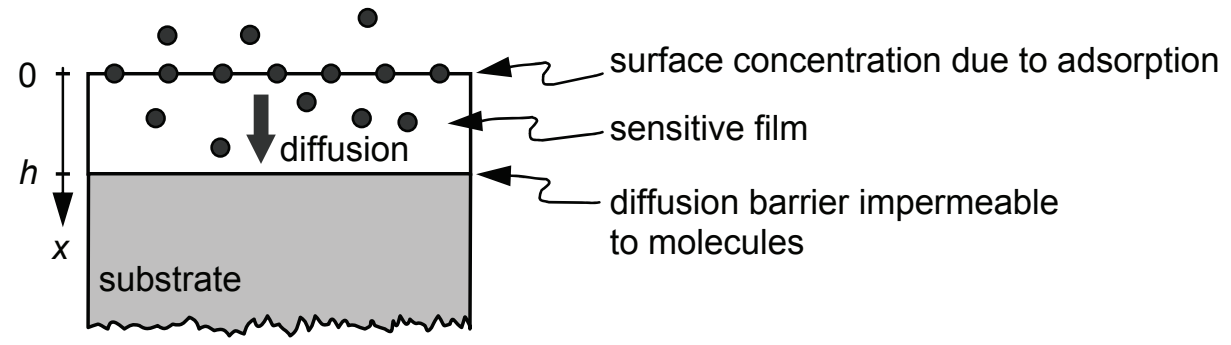

Fig. 5: Adsorption and diffusion of molecules in the sensor film of a SAW chemical sensor. 
The method can be extended to cover the full-fledged reaction-diffusion problem. This is accomplished by spatially discretizing the reaction-diffusion equations. This transforms the equations into a state-space form with the gas concentrations at equidistant locations inside the sensitive layer acting as state variables [13]. Standard signal-processing methods such as Kalman filtering and Wiener deconvolution can be readily applied on the basis of this state-space model.

\subsection{Comparison of methods}

The comparison of the previously discussed methods reveals that the FE and partial-wave methods are best suited for the investigation of basic functional dependences, that the delta-function model and equivalent-circuit methods are mostly useful for the computation of frequency characteristics and terminal impedances, and that perturbation approaches and state-space models are ideal for the quantification of the sensing effect proper (Table 1).

\begin{tabular}{|c|c|c|c|}
\hline Method & Advantages & Disadvantages & Use \\
\hline FE method & $\begin{array}{l}\text { - Flexibility } \\
\text { - Arbitrary geometries } \\
\text { - Handles SAW propagation, } \\
\text { excitation, detection, and } \\
\text { scattering }\end{array}$ & $\begin{array}{l}\text { - } \text { CPU time } \\
\text { Requires knowledge of all material } \\
\text { parameters (tensors) } \\
\text { - Must know dependence of material } \\
\text { parameters on } C_{a}\end{array}$ & $\begin{array}{l}\text { - Investigation of basic } \\
\text { functional dependences }\end{array}$ \\
\hline $\begin{array}{l}\text { Partial-wave } \\
\text { method }\end{array}$ & - Fast, straightforward & $\begin{array}{l}\text { - Only handles simple geometries } \\
\text { and undisturbed SAW propagation } \\
\text { - Must know dependence of material } \\
\text { parameters on } C_{a}\end{array}$ & $\begin{array}{l}\text { - Investigation of basic } \\
\text { functional dependences } \\
\text { - Search for special sub- } \\
\text { strate cuts }\end{array}$ \\
\hline $\begin{array}{l}\text { Delta-func- } \\
\text { tion model }\end{array}$ & - Fast, straightforward & $\begin{array}{l}\text { - Neglects important device effects } \\
\text { (e. g., finger reflectivity) } \\
\text { - No link to sensor effect }\end{array}$ & $\begin{array}{l}\text { - Determination of fre- } \\
\text { quency characteristics }\end{array}$ \\
\hline $\begin{array}{l}\text { Equivalent- } \\
\text { circuits }\end{array}$ & $\begin{array}{l}\text { - Easy to implement } \\
\text { - Model parameters unambi- } \\
\text { guously linked to device } \\
\text { characteristics }\end{array}$ & $\begin{array}{l}\text { - Dependence of model parameters } \\
\text { on } c_{a} \text { unclear } \\
\text { - Circuit modifications required for } \\
\text { coated or multi-layer devices }\end{array}$ & $\begin{array}{l}\text { - Determination of terminal } \\
\text { impedance }\end{array}$ \\
\hline $\begin{array}{l}\text { Perturbation } \\
\text { approach }\end{array}$ & $\begin{array}{l}\text { Directly relates measured } \\
\text { signal feature (e.g., } \\
\text { resonator frequency) to } c_{a}\end{array}$ & $\begin{array}{l}\text { - Predicts steady-state response only } \\
\text { - No link between model and device } \\
\text { design details }\end{array}$ & $\begin{array}{l}\text { Investigation of steady- } \\
\text { state sensor response }\end{array}$ \\
\hline $\begin{array}{l}\text { State-space } \\
\text { description }\end{array}$ & $\begin{array}{l}\text { - Directly relates measured } \\
\text { signal feature to } c_{a} \\
\text { - Only method to predict time } \\
\text { response of sensor }\end{array}$ & $\begin{array}{l}\text { No link between model and device } \\
\text { design details } \\
\text { Ambiguity between measurable } \\
\text { device characteristics and reaction- } \\
\text { diffusion equation parameters }\end{array}$ & $\begin{array}{l}\text { - Investigation of sensor } \\
\text { dynamics } \\
\text { - Signal processing for } \\
\text { device in field use }\end{array}$ \\
\hline
\end{tabular}

Table 1: Properties of selected SAW chemical sensor modeling methods. $c_{a}=$ analyte gas concentration.

\section{Signal processing approaches}

Even well-designed sensors exhibit undesired characteristics such as temperature sensitivity, slow response behavior, or noise. Some of these non-idealities can be remedied by hardware or by signal processing methods, provided that appropriate models for the sensing mechanism are available. If independent signal features can be extracted from the sensor terminal characteristics, this may be used to separate wanted effects from disturbances due to, e. g., the temperature. This was demonstrated for a $\mathrm{WO}_{3}$-coated 433-MHz SAW two-port resonator where temperature changes and analyte gas (hydrogen) concentration changes led to different trajectories in a signal-feature plane spanned by the resonance frequency $f_{\text {res }}$ and the return loss at resonance $\left|S_{11}\left(f_{\text {res }}\right)\right|[12]$.

By way of another example, let us consider the problem of the effective response time of a SAW chemical sensor. Owing to slow diffusion processes, it may take from seconds to hours until the steady-state frequency change is reached after an analyte gas concentration step. However, using a state-space model, one can estimate the steady-state value quite fast after the concentration step. To suppress the influence of measurement noise, the estimator can be implemented as Kalman filter which continuously compares the model prediction of the instantaneous sensor signal with the noisy measured signal and accordingly adjusts the model parameters, among which is the ambient gas concentration. 


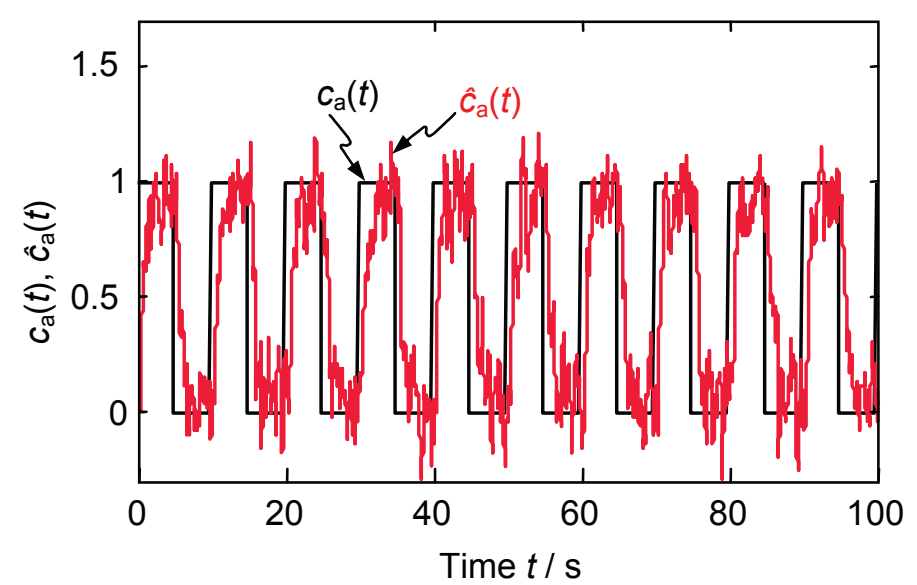

Fig. 6: Numerical experiment with time-varying ammonia concentration $c_{a}(t)$ and estimate $\hat{c}_{\mathrm{a}}(t)$ produced by Wiener deconvolution of the output signal of a state-space model for a SAW ammonia sensor corrupted by noise $(S N R=22 \mathrm{~dB})$. The settling time $t_{\mathrm{s}}$ of the modeled sensor, defined by $\Delta f\left(t_{\mathrm{s}}\right)=0,95 \cdot \Delta f_{\infty}$, was on the order of $3000 \mathrm{~s}(!)$, but the estimator predicts the steady-state value within about $5 \mathrm{~s}$ [13].

The same purpose is served by Wiener deconvolution, and it has been shown that such an approach is able to speed up the effective sensor response by one or more orders of magnitude even in the presence of noise (Fig. 6).

\section{Conclusion}

This contribution has discussed the salient features of various SAW device models and their possible use in the context of chemical sensors. It was demonstrated that they have their respective strengths and weaknesses. It is believed that the characteristics of SAW chemical sensor systems, determined by the sensor element, the signal conditioning circuit, and the signal processing, is far from optimum up to now. A better use of system-level methods such as signal processing should allow one to improve on the state of the art in a way similar to the one demonstrated in Section 3 for the effective response time.

\section{References}

[1] B. A. Auld, Acoustic Fields and Waves in Solids. Vol. I, II. Malabar: Krieger Publishing, 1990.

[2] G. Sauerbrey, "Verwendung von Schwingquarzen zur Wägung dünner Schichten und zur Mikrowägung" (in German), Zeitschrift für Physik 155, pp. 206-222, 1959

[3] E. Hymon, "Finite-Elemente-Simulation Piezoelektrischer Mikrosysteme" (in German), Diploma Thesis, Bayreuth, 2006

[4] D. P. Morgan, Surface-Wave Devices for Signal Processing. Amsterdam u. a.: Elsevier, 1991.

[5] W. Ruile, "P-Matrix-Modell zur Simulation von Oberflächenwellenbauelementen" (in German), Ph. D. Thesis, Munich, 1994

[6] J. F. Rosenbaum, Bulk Acoustic Wave Theory and Devices. Boston, London: Artech House, 1988.

[7] H. F. Tiersten, "Elastic surface waves guided by thin films," J. Appl. Physics, Vol. 40, pp. 770-789, 1969

[8] G. Fischerauer, "Acoustic surface wave devices," in: W. Göpel et al. (eds.), Sensors. A comprehensive survey. Vol. 8. Weinheim: VCH, 1995, pp. 135-157.

[9] D. S. Ballantine, R. M. White, S. J. Martin, A. J. Ricco, E. T. Zellers, G. C. Frye, H. Wohltjen, Acoustic Wave Sensors : Theory, Design, and Physico-Chemical Applications. San Diego etc.: Academic Press, 1997.

[10] P. N. Bartlett, J. W. Gardner, "Diffusion and binding of molecules to sites within homogeneous thin films," Phil. Trans. R. Soc. Lond. A, Vol. 354, pp. 35-57, 1996

[11] G. Fischerauer, F. Dickert, "An Analytic Model of the Dynamic Response of Mass-Sensitive Chemical Sensors," Sensors and Actuators B, Vol. 123, No. 2, pp. 993-1001, May 2007

[12] M. Adam, G. Fischerauer, "Trennung von Temperatur- und Analyteinflüssen bei massensensitiven Chemosensoren” (in German), Dresdner Beiträge zur Sensorik, Bd. 39, pp. 329-332, Dec. 7-9, 2009

[13] F. Thalmayr, G. Fischerauer, "Sensor signal processing for gravimetric chemical sensors based on a state-space model," Sensors and Actuators B, Vol. 144, No. 1, pp. 27-36, Jan. 2010 